\title{
Characterization of focal liver lesions using sulphur hexafluoride (SF6) microbubble contrast-enhanced ultrasonography
}

\author{
Mohamed Gamal El-Din Mansour ${ }^{1}$, Mona Hussein Abdel Kader ${ }^{2}$, Hanan Mahmoud Arafa ${ }^{1}$ and Susan Adil Ali ${ }^{*}$
}

\begin{abstract}
Background: Focal hepatic lesions incidentally detected during ultrasound usually need further step for proper characterization. The aim of this study was to highlight the efficacy of microbubble contrast-enhanced ultrasonography (CEUS) in characterization of focal liver lesions. This prospective study was conducted on 60 patients presented with hepatic focal lesions in the period from January 2019 to June 2020. CEUS studies were performed after a baseline conventional ultrasound with the same machine by the same operator. The ultrasound contrast agent used is second-generation US contrast agent. The enhancement patterns of the hepatic lesions were studied during the vascular phases up to $5 \mathrm{~min}$ and the data were correlated with histopathology, triphasic contrast-enhanced CT, and clinical follow-up.

Results: CEUS demonstrated a sensitivity of $94.2 \%$, specificity of $88.9 \%$, positive predictive value of $91 \%$, negative predictive value of $94.1 \%$, and accuracy of $92.3 \%$ for characterization of hepatic focal lesions, compared to a sensitivity of $100 \%$, specificity of $81.8 \%$, positive predictive value of $84 \%$, negative predictive value of $100 \%$, and accuracy of $90.7 \%$ for triphasic CT.
\end{abstract}

Conclusion: CEUS is an effective tool in characterization of HFLs and recommended as a second diagnostic step after conventional ultrasound to immediately establish the diagnosis especially in patients with contraindications to CECT.

Keywords: Contrast-enhanced ultrasonography, Microbubble contrast agents, Liver focal lesions

\section{Background}

Focal liver lesions are usually detected incidentally during an abdominal ultrasound examination, during surveillance in chronic liver diseases and cirrhosis or during first evaluation or follow-up for a primary neoplasm, but the accuracy of the final definitive diagnosis can be limited [1]. In fact, even though color Doppler imaging during ultrasound study of the liver can improve diagnostic confidence in the characterization of focal liver lesions, it has important limitations because of limited sensitivity and specificity because benign and malignant lesions may show similar appearance on B-mode and Doppler

\footnotetext{
* Correspondence: dr.susanadil@hotmail.com

${ }^{1}$ Radiodiagnosis Department, Ain Shams University, Cairo, Egypt

Full list of author information is available at the end of the article
}

ultrasound [2]. Further examinations as CT, MRI, or PET CT can be used for characterization and posttherapeutic follow-up of HFLs; however, each examination has its own limitations and consequently CEUS is considered a more easy, safe, rapid, and accurate alternative [3-6]. CEUS is a novel imaging technique using microbubble contrast agents that has approved by many countries to improve the detection and characterization of focal liver lesions by obtaining the real-time enhancement of lesions [7-9]. Although CEUS is subjected to the same limitations as ordinary US and is inferior to CECT/CEMRI in some aspects, CEUS has proved to be of great value in the management of HCC with inherent advantages, such as sufficient high safety profile making it suitable for patients with renal failure or allergic to
Springer Open (c) The Author(s). 2021 Open Access This article is licensed under a Creative Commons Attribution 4.0 International License, which permits use, sharing, adaptation, distribution and reproduction in any medium or format, as long as you give appropriate credit to the original author(s) and the source, provide a link to the Creative Commons licence, and indicate if changes were made. The images or other third party material in this article are included in the article's Creative Commons licence, unless indicated otherwise in a credit line to the material. If material is not included in the article's Creative Commons licence and your intended use is not permitted by statutory regulation or exceeds the permitted use, you will need to obtain permission directly from the copyright holder. To view a copy of this licence, visit http://creativecommons.org/licenses/by/4.0/. 
iodine, absence of radiation, easy reproducibility, and high temporal resolution [10]. The use of CEUS is recommended in official guidelines and suggested as a second diagnostic step after ultrasound detection of indeterminate focal liver lesions to immediately establish the diagnosis, especially for benign liver lesions, such as hemangiomas, avoiding further and more expensive examinations [11]. The purpose of the study was to evaluate the efficacy of contrast-enhanced ultrasonography in characterization of hepatic focal lesions.

\section{Methods}

\section{Patients}

This was a prospective study conducted on 60 patients with hepatic focal lesions from January 2019 to June 2020, included 38 males (63.3\%) and 22 female (36.7\%) ranging in age between 32 and 68 years with mean age of $53.83 \pm 10.664$. Inclusion criteria include age between 18 and 70 years, incidental detection of hepatic focal lesion on sonography, patients with cirrhosis being evaluated for hepatocellular carcinoma or post-therapeutic follow-up and suspected liver metastasis. Exclusion criteria include age less than 18 years, pregnant or lactating women, acoustic window insufficient for adequate sonographic examination of the liver, critically ill or medically unstable patients, known allergy to any component of US contrast agent. The present study was approved by the institutional review board and all patients were informed about the study and provided written informed consents.

\section{Technique}

\section{Conventional B-mode and color Doppler scanning}

Ultrasonography was performed using a real-time machine (Hitachi, EUB-7500-Hitachi Medical Systems, Japan) with a $3.5 \mathrm{MHz}$ convex array probe.

\section{Contrast-enhanced ultrasound (CEUS)}

CEUS studies were performed after a baseline ultrasound with the same machine used in conventional ultrasound. CEUS was performed by the same operator (has 15 years of experience) with a preinstalled contrastspecific sonographic imaging mode (a low frame rate (5 $\mathrm{Hz})$ and a very low mechanical index $(\mathrm{MI})<0.08$, were used for real-time imaging). The ultrasound contrast agent UCA used is 2nd generation US contrast agent SonoVue (Bracco, Italy). SonoVue is a kit including one vial [containing $25 \mathrm{mg}$ of lyophilized powder the active substance is sulphur hexafluoride in the form of microbubbles, macrogol4000, distearoyl phosphatidyl choline, dipalmitoyl phosphatidyl glycerol sodium, and palmitic acid], one pre-filled syringe containing $5 \mathrm{ml}$ sodium chloride $0.9 \%$ and one Mini-Spike transfer system. The microbubble dispersion is prepared before use by injecting $5 \mathrm{ml}$ of sodium chloride $0.9 \%$ solution to the contents of the vial. The vial is then shaken for a few seconds until the lyophilisate is completely dissolved, and a homogeneous white milky liquid is obtained. CEUS studies were carried out after the administration of $2.4 \mathrm{ml}$ of the SonoVue (for each lesion to be characterized) as a bolus via a 20 gauge peripheral intravenous cannula, followed by a $10 \mathrm{ml}$ saline flush. The injection was repeated using the same dose $(2.4 \mathrm{ml})$ or double dose $(4.8 \mathrm{ml})$, up to a total dose of $9.6 \mathrm{ml}$ for multilesion assessment if needed. All patients were monitored for adverse events, for $4 \mathrm{~h}$ after the procedure. The clinical status, blood pressure, and heart rate were followedup, yet no adverse events occurred in any patient.

\section{Image interpretation}

Complete assessment of the liver by conventional Bmode scanning was done with special focus on focal lesions assessing the number, size, site, the echogenicity of the focal lesion. Portal vein diameter and patency were assessed using color and power Doppler. Splenic size and texture as well as presence of ascites were assessed. Any abdominal masses or lymph node enlargement were also commented upon. By CEUS, the enhancement patterns of the hepatic lesions were studied during the vascular phases up to $5 \mathrm{~min}$, including the arterial (10-45 s), portal (45-120 s), and late phases (120-300 s). All sonographic examinations were digitally recorded. A CEUS examination was considered conclusive if following contrast administration, the focal lesion had a typical enhancement pattern and no other diagnostic methods were required, while considered inconclusive if the enhancement pattern of the lesions was not typical and correlation with other diagnostic methods were performed (contrast CT or biopsy of the lesion).

All the studied cases with metastasis, adenomas, and few cases of HCCs were diagnosed by biopsy. Diagnosis of rest of cases was done by correlation between patient history, available triphasic CT study, serum AFP particularly in patients with post-therapeutic recurrence, and malignant PVT as well as patient follow up.

\section{Statistical analysis}

Results are expressed as mean \pm standard deviation or number (\%). Comparison between categorical data was performed using chi-square test. Standard diagnostic indices including sensitivity, specificity, positive predictive value (PPV), negative predictive value (NPV), and diagnostic efficacy were calculated as described by (Galen, 1980). SPSS computer program (version 12 Windows) was used for data analysis. $P$ value less than or equal to 0.05 was considered significant. 


\section{Results}

Of total 60 patients included in the study, 30 patients had HCCs (22 males and 8 females). Among them, 18 patients underwent therapeutic intervention (8 patients underwent RFA, 8 patients underwent TACE, and 2 patients underwent ethanol injection).

Regarding the number of lesions in the HCC patients by triphasic CT; 36 lesions were found, 26 patients had 1 lesion, 2 patients had 2 lesions, and 2 patients had 3 lesions. Fourteen lesions (39\%) were HCC, 12 lesions (33.3\%) were ablated HCC, 8 lesions (22.2\%) were incompletely ablated HCC, and 2 lesions (5.5\%) were HCC with malignant PVT.24 (66.7\%) lesions were located in the right lobe, 12 (33.3\%) were located in the left lobe. The mean size of the lesions was $28.21 \pm 12.242 \mathrm{~mm}$. The portal vein was patent in $22(77.3 \%)$ patients and thrombosed in $8(26.7 \%)$ patients.

Regarding the number of lesions in the HCC patients by CEUS; 34 lesions were found. Twenty-six patients had 1 lesion and 4 patients had 2 lesions. Twelve lesions (35.3\%) were HCC, 12 lesions (35.3\%) were ablated HCC, 8 patients (23.5\%) were incompletely ablated HCC, and 2 patients (5.9\%) were HCC with malignant PVT (Fig. 1). Twenty-four lesions (66.7\%) were located in the right lobe, 10 lesions (27.8\%) were in the left lobe, and 2 lesions (5.5\%) had not detected by CEUS. The mean size of the lesions was $28.97 \pm 12.864 \mathrm{~mm}$.

Regarding the echogenicity of HCC lesions using conventional B-mode, 4 lesions (11.76\%) were isoechoic, 22 lesions $(64.7 \%)$ were hypoechoic, 4 lesions $(11.76 \%)$ were hyperechoic, and 4 lesions (11.76\%) were heterogenous. After intravenous injection of SonoVue, 22 lesions (64.7\%) were hyper-enhanced [of which 14 lesions (41.2\%) showed homogenous enhancement and 8 lesions (23.5\%) showed eccentric nodular enhancement with mean size of $16 \pm 4 \mathrm{~mm}$ ] and 12 lesions (53.3\%) were non-enhanced in the arterial phase. In the portal phase, 14 lesions (41.2\%) were iso-enhanced, 8 lesions (23.5\%) were hypo-enhanced, and 12 lesions (35.3\%) were nonenhanced. In the late phase, 22 lesions (64.7\%) were hypo-enhanced and 12 lesions (35.3\%) were nonenhanced. No lesions were enhanced in the portal/late phases. The portal vein was patent in $22(77.3 \%)$ patients and thrombosed in 8 (26.7\%).

CEUS showed typical HCC enhancement pattern in 30 (88.2\%) lesions; 14 lesions were active HCCs (Fig. 2), 8 lesions were ablated HCCs (Fig. 3) and 8 lesions were incompletely ablated HCCs. CEUS showed atypical enhancement pattern in $4(11.8 \%)$ incompletely ablated lesions which CEUS misdiagnosed as ablated. There was no statistically significant difference $(p$ value $>0.05)$ between CEUS and triphasic CT in the characterization of HCCs (Table 1). CEUS detected PVT in 8 patients $(100 \%)$ and was able to characterize them, where 6 (75\%) were bland non enhancing thrombi and $2(25 \%)$ were pathologically enhancing thrombi being homogenously enhancing in arterial phase and showing rapid washout in portal and delayed phases.

A total of 12 patients were diagnosed with liver metastases, with one lesion was studied in each patient. Triphasic CT abdomen and CEUS were done to all patients. The mean age of patients with liver metastasis was $50.33 \pm 12.61$ years. Six patients $(50 \%)$ were females, and 6 patients $(50 \%)$ were males. The pattern of CT enhancement of the metastatic lesions (biopsy proven) was as follows: 6 hypervascular metastases (50\%), which were metastatic renal cancer, melanoma, and choriocarcinoma, 4 hypovascular metastases $(33.3 \%)$ from rectal and ovarian cancers, and 2 post-therapeutic metastases (16.7\%) from pathologically proven neuroendocrine

\section{Diagnosis in HCC patients}

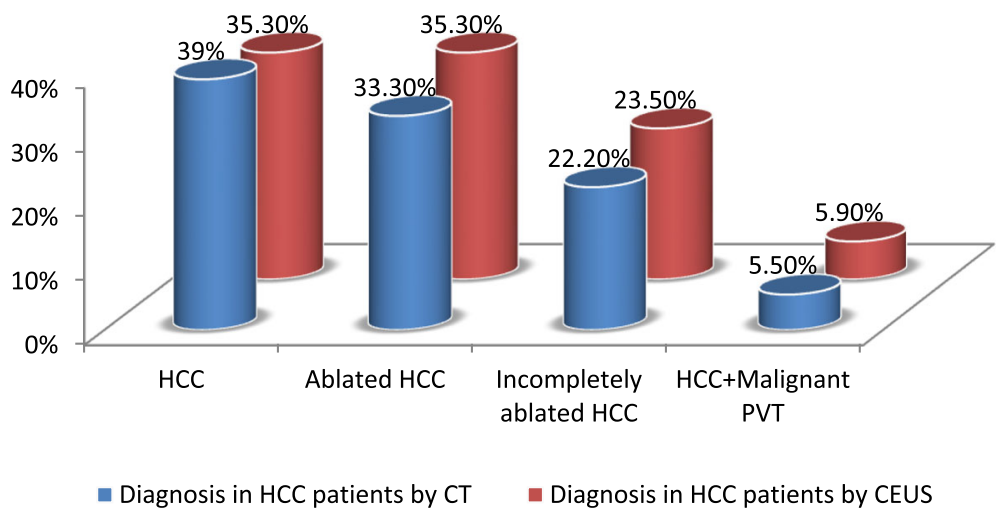

Fig. 1 Diagnosis and post-therapeutic assessment of HCCs by CT and CEUS 

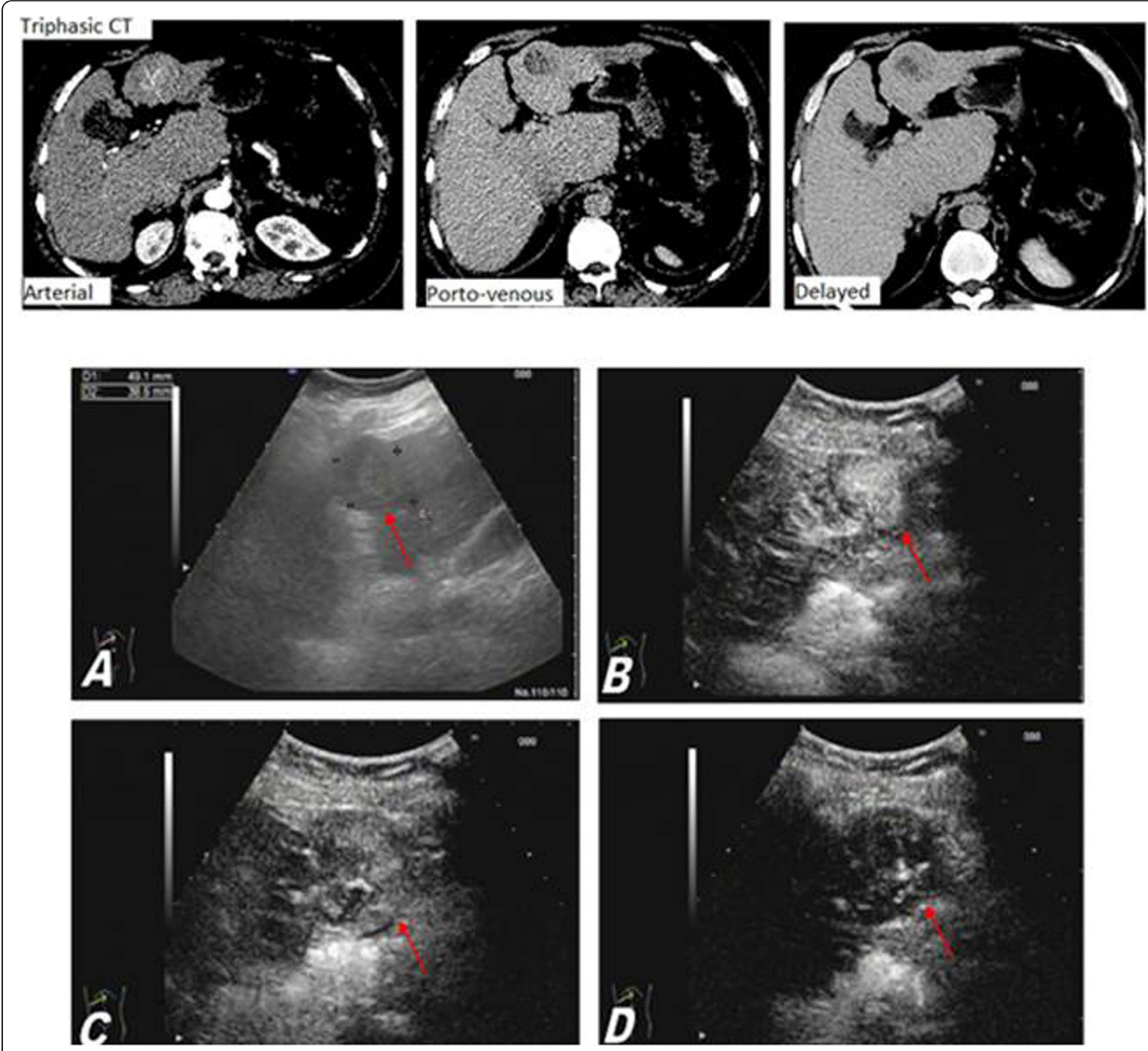

Fig. 2 A 59-year-old female, HCV + ve with elevated AFP serum level (340 ng/ml) and accidentally discovered HFL on routine US check-up. Triphasic CT examination reveals a left lobe exophytic lesion with arterial phase enhancement followed by washout in the porto-venous and delayed phases. a NEUS revealed an exophytic hypoechoic focal lesion. b-d CEUS real-time examination during early arterial, portal, and late phases showed early homogenous arterial contrast uptake with rapid washout to appear hypoechoic in late phase, consistent with HCC

tumors. The 12 lesions were identified by CT; 6 lesions $(50 \%)$ in the right hepatic lobe and 6 lesions $(50 \%)$ in the left lobe and mean size of the lesions was $28.83+17.279$ $\mathrm{mm}$. Regarding CEUS, the 12 lesions were identified; 6 lesions (50\%) in the right lobe and 6 lesions $(50 \%)$ in the left lobe. The mean size of the lesions was $29.21+$ $16.819 \mathrm{~mm}$. Regarding the echogenicity of lesions using conventional B-mode, 4 lesions (33.3\%) were hypoechoic and 8 lesions (66.7\%) were isoechoic. After intravenous injection of SonoVue, 6 lesions (50\%) showed complete hyper enhancement (hypervascular metastases), 4 lesions (33.3\%) showed no enhancement (hypovascular metastases) (Fig. 4) and 2 lesions (16.7\%) showed rim enhancement in the arterial phase (post-therapeutic metastasis). In the portal and late phases, all lesions (100\%) were non-enhanced. CEUS showed typical enhancement pattern in the $12(100 \%)$ lesions. There was no statistically significant difference $(p$ value $>0.05)$ between CEUS and CT in the characterization of hepatic metastases (Table 2).

Eighteen patients (8 females and 10 males) were diagnosed with 22 benign hepatic focal lesions by triphasic $\mathrm{CT}$, and their mean age was $44 \pm 8.047$ years. Twelve lesions $(54.5 \%)$ were located in the right lobe and 10 

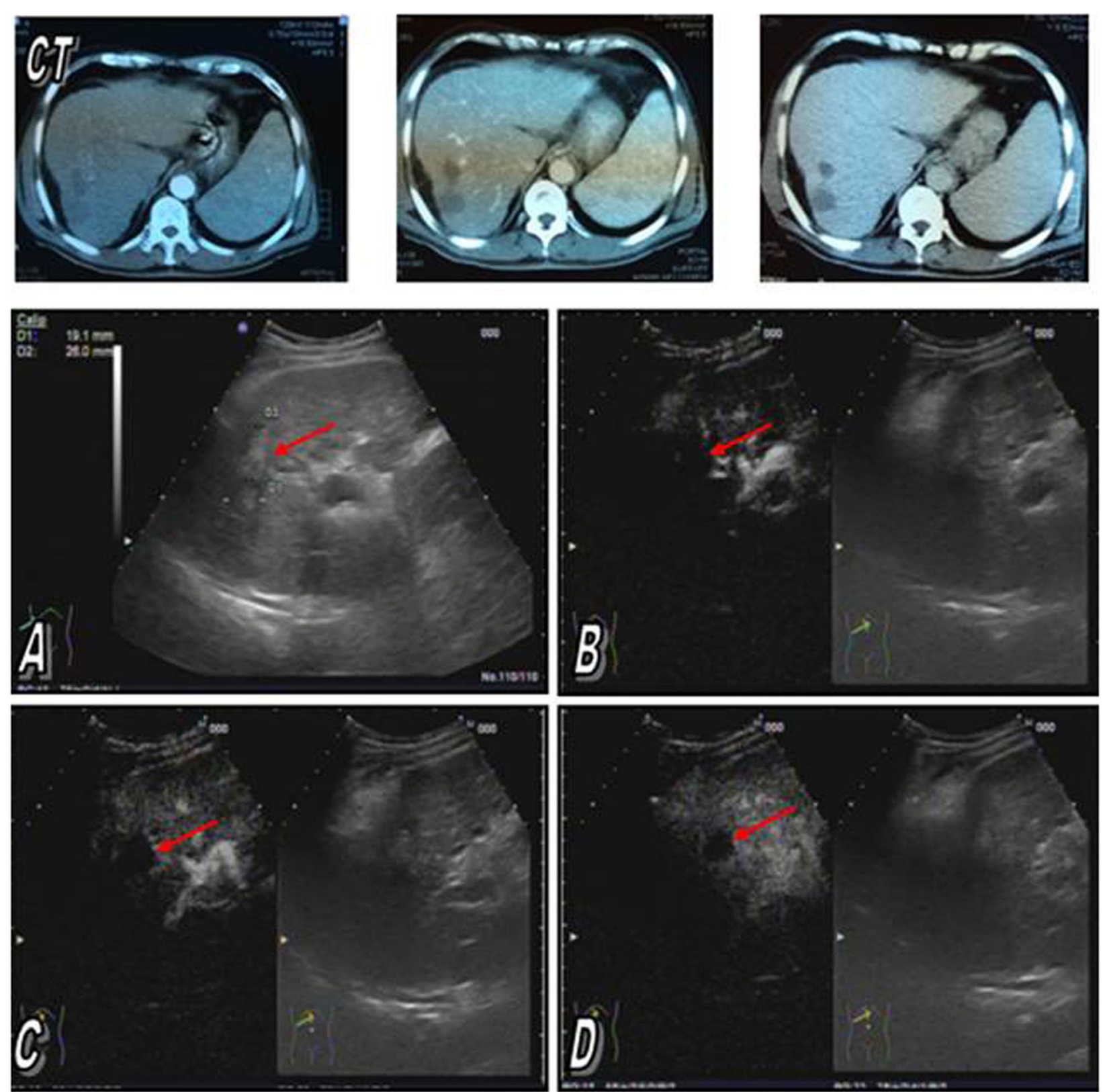

Fig. 3 A 62-year-old male, HCV + ve, AFP serum level (4 ng/ml), referred for follow-up post-radiofrequency ablation. Triphasic CT study revealed persistent non-enhancing hypodense ablation zone within right hepatic lobe. a NEUS revealed two adjacent hyperechoic lesions measuring about $2.6 \mathrm{~cm}$ and $1.9 \mathrm{~cm}$. b-d CEUS real-time examination during early arterial phase, portal phase, and late phase showed persistent nonenhancement of the lesions, consistent with well-ablated HCC

Table 1 CECT and CEUS enhancement patterns showed in HCCs patients

\begin{tabular}{lllllll}
\hline Enhancement pattern & Typical & & & Atypical & & Total \\
\cline { 2 - 7 } & CECT & CEUS & CECT & CEUS & CECT & CEUS \\
\hline HCCs & 12 & 14 & 4 & 0 & 16 & 14 \\
Incompletely ablated HCCS & 8 & 8 & 4 & 4 & 12 & 12 \\
Ablated HCCs & 8 & 8 & 0 & 0 & 8 & 8 \\
Total & 28 & 30 & 8 & 4 & 36 & 34 \\
\hline
\end{tabular}



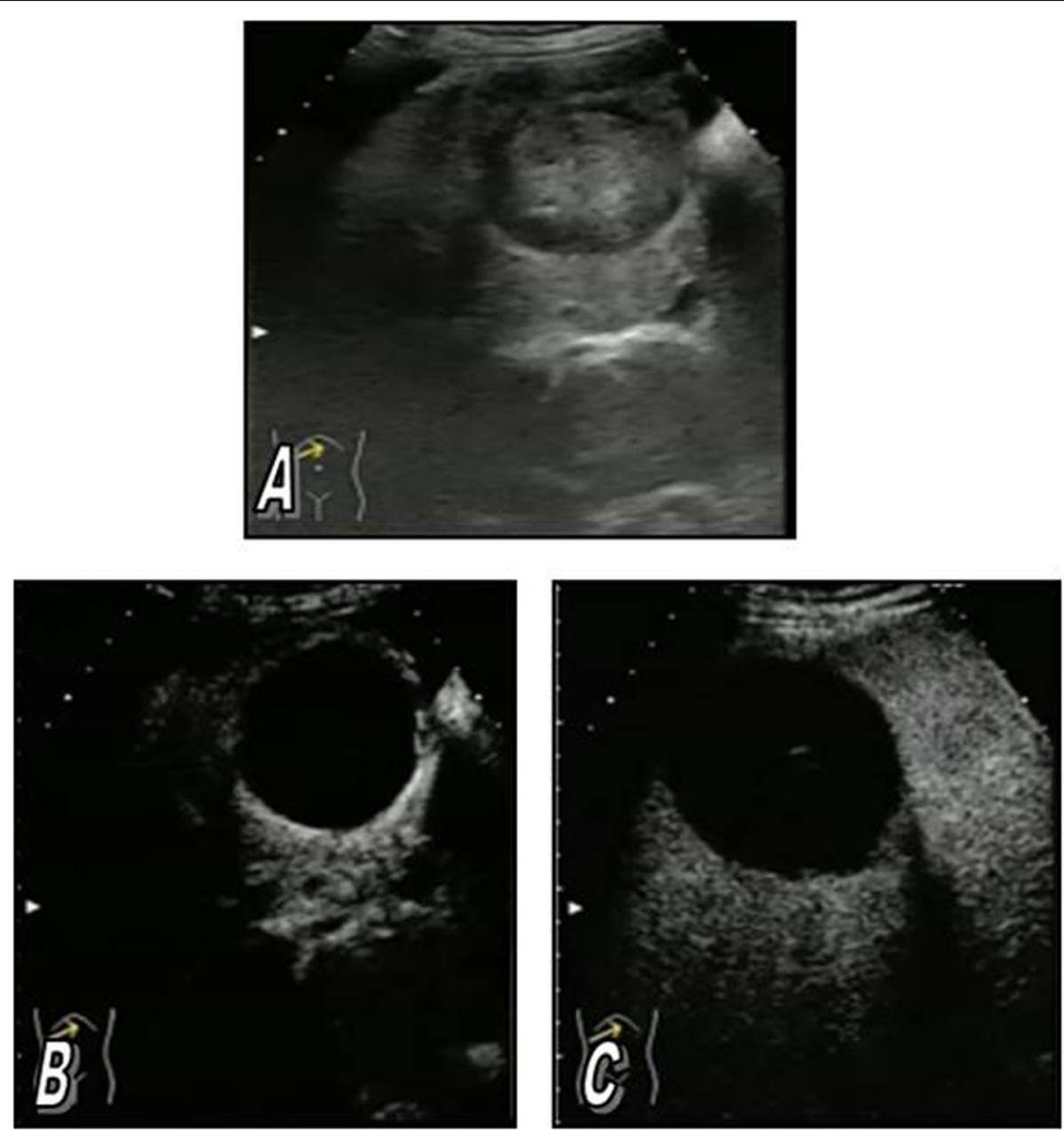

Fig. 4 A 58-year-old female, HCV -ve with history of pathologically proven ovarian cancer. a NEUS revealed average-sized liver showing left lobe segment IV isoechoic lesion measuring about $6.3 \mathrm{~cm}$. CEUS revealed rim enhancement in the arterial phase (b) and the lesion is non-enhancing in late phase (c), suggesting hypovascular metastasis

lesions (45.5\%) were in the left lobe. The mean size of the lesions was $39.22+20.191 \mathrm{~mm}$. The diagnosis of the lesions was as follows: 16 hemangiomas (from which 2 were undetectable by CEUS), 2 adenomas, 2 regeneration nodules, and 2 hydatid cysts. By CEUS, 20 lesions were identified and 2 lesions could not be identified; 10 lesions (45.5\%) in the right lobe and 10 lesions (45.5\%) in the left lobe. The mean size of the lesions was $40.02+$ $20.233 \mathrm{~mm}$. Regarding the echogenicity of the lesions by conventional B-mode, 6 lesions (30\%) were hyperechoic,
2 lesions (10\%) were isoechoic, 6 lesions (30\%) were heterogenous, and 6 lesions (30\%) were hypoechoic. After intravenous injection of SonoVue, 2 lesions (10\%) showed homogenous enhancement, 10 lesions (50\%) showed peripheral nodular enhancement, and 8 lesions (40\%) showed no enhancement in the arterial phase. In the portal phase, 2 lesions (10\%) showed homogenous enhancement, 10 lesions (50\%) showed centripetal filling in enhancement, 4 lesions (20\%) showed no enhancement, and $4(20 \%)$ lesions were iso-enhanced. In the late

Table 2 CECT and CEUS enhancement patterns showed in patients with hepatic metastases

\begin{tabular}{lllllll}
\hline Enhancement pattern & Typical & & & Atypical & & Total \\
\cline { 2 - 7 } & CECT & CEUS & CECT & CEUS & CECT & CEUS \\
\hline Hypervascular metastases & 4 & 6 & 2 & 0 & 6 & 6 \\
Hypovascular metastases & 4 & 4 & 0 & 0 & 4 & 4 \\
Post-therapeutic metastases & 2 & 2 & 0 & 0 & 2 & 2 \\
Total & 10 & 12 & 2 & 0 & 12 & 12 \\
\hline
\end{tabular}


phase, 6 lesions (30\%) showed homogenous enhancement, 4 lesions (20\%) showed incomplete enhancement with non-enhancing cores, 6 lesions (30\%) were isoenhanced, and 4 lesions (20\%) were non-enhanced. No statistically significant difference was found in the findings between CEUS and triphasic CT ( $p$ value $>0.05$ ).

Regarding the enhancement pattern of triphasic CT scan in the diagnosis of benign hepatic focal lesions, CT showed typical enhancement pattern in all $22(100 \%)$ lesions [16 hemangiomas, 2 adenomas, 2 regeneration nodules, and 2 hydatid cysts]. CEUS showed typical enhancement pattern in 18 lesions [12 lesions were hemangiomas, 2 regeneration nodules, 2 adenomas (Fig. 5) and 2 hydatid cysts (Fig. 6)]. CEUS showed atypical enhancement pattern in 2 lesions which were hemangiomas. There was no statistically significant difference ( $p$ value > 0.05) between CEUS and triphasic CT in the characterization of benign hepatic focal lesions (Table 3).

Triphasic CT demonstrated a sensitivity of $100 \%$, specificity of $81.8 \%$, positive predictive value of $84 \%$, negative predictive value of $100 \%$, and accuracy of $90.7 \%$, while CEUS demonstrated a sensitivity of $94.2 \%$, specificity of $88.9 \%$, positive predictive value of $91 \%$, negative predictive value of $94.1 \%$, and accuracy of $92.3 \%$ for characterization of hepatic focal lesions.

\section{Discussion}

CEUS has improved the characterization of focal liver lesions showing comparable results to those with CT and MRI and when performed by experienced operators, it significantly improves overall diagnostic accuracy by more than $30 \%$ compared with unenhanced ultrasound [12].

This study was conducted on 60 patients with 70 hepatic focal lesions. CEUS and triphasic CT were done to all patients. No adverse events occurred after the administration of SonoVue. From 70 focal lesions assessed, CEUS missed 4 lesions (2 HCCs and 2 hemangiomas). That was due to either very small size or deeply situated lesions or those lesions seated within hepatic dome hindered by the costal margin and was not easily accessible. Thus, in this study that investigated the role of CEUS in characterization of malignant from benign hepatic focal lesions, the sensitivity, specificity, PPV, NPV, and accuracy of CEUS in the differentiation between benign and malignant hepatic focal lesions were $94.2 \%, 88.9 \%, 91 \%$, $94.1 \%$, and $92.3 \%$, respectively, and for triphasic CT were $100 \%, 81.8 \%, 84 \%, 100 \%$, and $90.7 \%$, respectively. There was no statistically significant difference between CEUS and triphasic CT.

The two most important multi-center studies regarding CEUS application for the characterization of focal liver lesions were the German Society of Sonography
(DEGUM) multi-center study and a French study, showed good value for CEUS for focal liver lesion characterization [13, 14]. The DEGUM study included 1349 patients with focal liver lesions on ultrasound. A total of 1328 focal liver lesions (755 malignant and 573 benign) were assessed. The reference standard diagnosis was made by means of liver biopsy in $75 \%$ of cases and by contrast-enhanced CT or contrast-enhanced MRI in the other cases. The accuracy of CEUS for the diagnosis of focal liver lesions was 90.3\%. CEUS showed 95.8\% sensitivity and $83.1 \%$ specificity, with $95.4 \%$ positive predictive value and $95.9 \%$ negative predictive value for differentiating benign versus malignant lesions. The French study assessed the clinical value of CEUS using SonoVue for the characterization of focal liver lesions discovered in patients with a cancer history or in those with chronic liver disease. The study included 1034 focal liver lesions undiagnosed on ultrasound alone. The reference standard methods were contrast-enhanced $\mathrm{CT}$, contrastenhanced MRI, or liver biopsy and CEUS had 79.4\% sensitivity and $88.1 \%$ specificity in differentiating benign versus malignant focal liver lesions. These findings are also approximate to the study done by Sporea and Sirli, included 573 benign lesions and 755 malignant lesions, who investigated if CEUS ready for use in daily practice for evaluation of focal liver lesion. The overall accuracy of CEUS for the diagnosis of HFLs was $90.3 \%$. CEUS had $95.8 \%$ sensitivity and $83.1 \%$ specificity, with $95.4 \%$ positive predictive value (PPV) and $95.9 \%$ negative predictive value (NPV) for differentiating benign versus malignant lesions [15]. Another study by Trillaud et al. for characterization of focal liver lesions with SonoVue enhanced sonography in comparison to CT in which 68 focal liver lesions were benign and 55 were malignant showed sensitivity, specificity and accuracy of 95.5\%, $75.0 \%$, and $90.0 \%$ for CEUS and $72.7 \%, 37.5 \%$, and $63.3 \%$ for CT. In comparison, CT was significantly less sensitive $(p<0.0001)$, less specific $(p<0.029)$, and less accurate $(p<0.0001)$ than SonoVue enhanced ultrasound unlike our study [16]. Although our results using SonoVue enhanced ultrasound were near from this study regarding the sensitivity, specificity and accuracy, but we did not find any statistically significant difference between the different imaging modalities $(p<0.452)$.

In this study, CEUS using SonoVue correctly characterized 30 (83.3\%) HCC lesions, $16(72.7 \%)$ benign lesions, and $12(100 \%)$ metastatic lesions and showed atypical enhancement pattern in 4 (11.1\%) HCC lesions, 4 (18.2\%) benign lesions, and none of metastatic patients while CT correctly characterized 28 (77.8\%) HCC lesions, 22 (100 \%) benign lesions, and 10 (83.3\%) metastatic patients and showed atypical enhancement in 8 (22.2\%) HCC lesions, none of benign lesions, and 2(16.7\%) of metastatic lesions. Thus, there was no statistically 

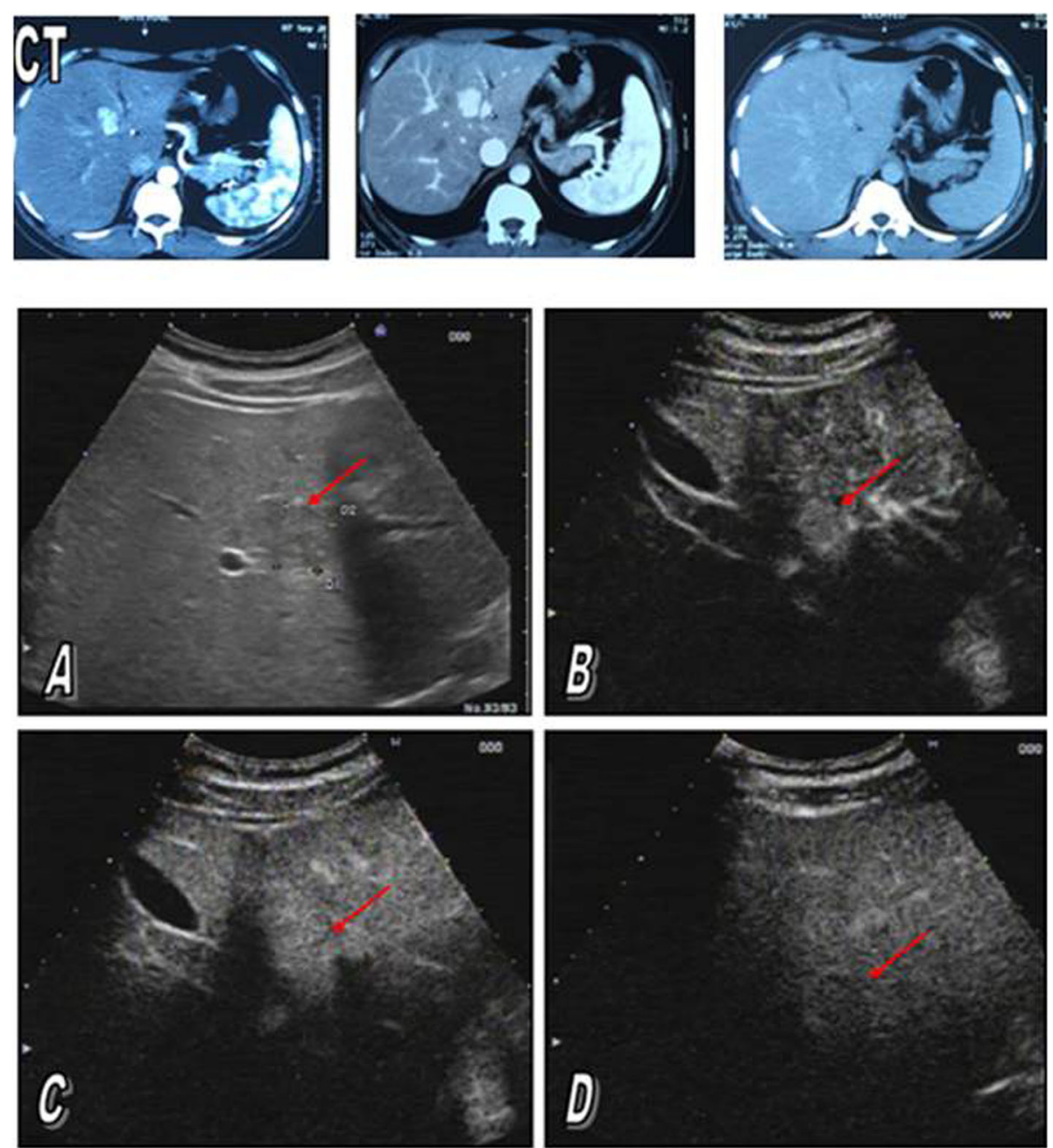

Fig. 5 A 32-years-old female with accidentally discovered focal lesion. Triphasic CT study revealed homogenously enhancing focal lesion in arterial and portal phases that became isodense to hepatic parenchyma in delayed phase. a NEUS revealed average sized liver showing segment IV hypoechoic focal lesion measuring about $2.3 \mathrm{~cm}$. b-d CEUS real-time examination of the focal lesion showed early homogenous contrast uptake that became iso to slightly hypoechoicin delayed phase, histopathologically proven to be hepatic adenoma

significant difference between both modalities. Our findings are in agreement with Martie et al. and Laroia et al. who studied the role of CEUS using SonoVue in the characterization of 100 and 50 patients with HCCs, respectively. CEUS using SonoVue correctly characterized $75.7 \%$ and $88 \%$ of the lesions, respectively $[17,18]$.

In this study, 8 patients with HCC had associated portal vein thrombosis, of which 2 were malignant thrombi. CEUS and triphasic CT detected and correctly characterized $8 / 8$ thrombi $(100 \%)$. There is no statistically significant difference between them. These findings are in disagreement with a study conducted by Rossi et al. who compared CEUS and triphasic CT in the detection and characterization of PVT complicating $\mathrm{HCC}$ in 50 patients, in which 44 thrombi were pathologically diagnosed as malignant and 6 were benign. CEUS detected $50 / 50(100 \%)$ thrombi and correctly characterized 49/50
(98\%) while CT detected 34/50 (68\%) thrombi and correctly characterized $23 / 34$ (68\%), So, CEUS outperformed triphasic CT in terms of both thrombus detection and characterization in this study [19].Another study by Sorrentino et al. who investigated CEUS versus biopsy for the differential diagnosis of PVT in 108 HCC patients, 58 patients (53.7\%) with malignant PVT and 50 (46.3\%) with benign PVT. Sensitivity, specificity, positive and negative predictive value of biopsy and CEUS were the same for both: $89.6 \%, 100 \%, 100 \%$ and $89.2 \%$, respectively [20].

Regarding the role of CEUS in the assessment of HCC after therapeutic intervention, this study showed that CEUS correctly identified 8 (40\%) ablated HCC and 8 incompletely ablated HCC (40\%) but misdiagnosed 4 incompletely ablated HCC (20\%) as ablated. These findings are in agreement with a multi-center study by 

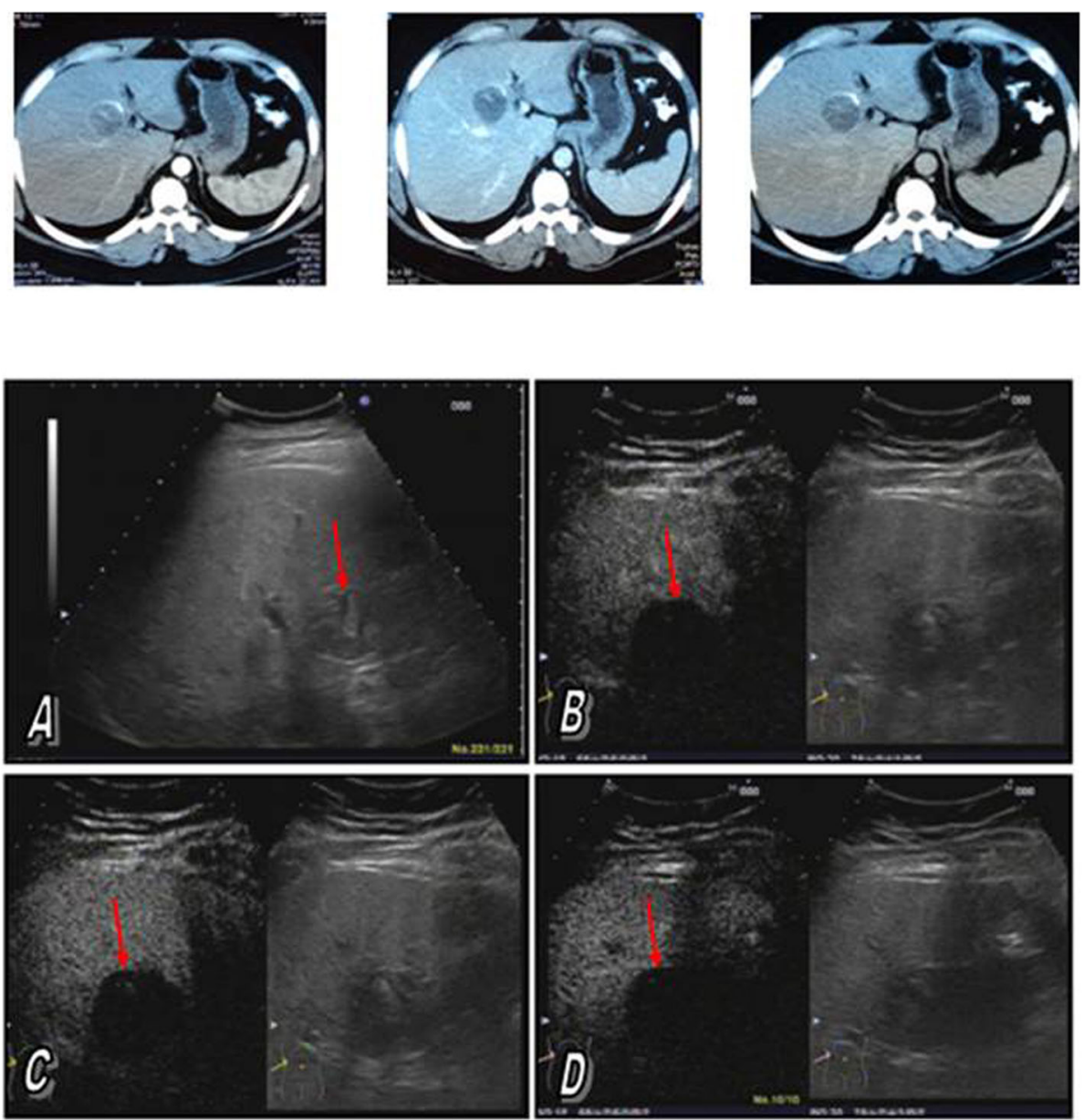

Fig. 6 A 36-year-old male, known case of hydatid disease. Triphasic CT study revealed left lobe segment IV cystic non-enhancing lesion with marginal calcification. a NEUS revealed average-sized liver showing left lobe segment IV heterogeneous cystic lesion measuring about $4 \mathrm{~cm}$. $\mathbf{b}-\mathbf{d}$ CEUS real-time examination during arterial phase, portal phase, and late phase showed persistent non-enhancement

$\mathrm{Lu}$ et al. to evaluate the ability of CEUS using SonoVue in monitoring percutaneous thermal ablation procedure in patients with HCC in comparison with contrastenhanced CT and/or MRI. One hundred eighteen patients were monitored to assess the tumor response to treatment within 1 month after the ablation procedure by radiofrequency or microwave ablation. No enhancement was seen in 110/118 (93.2\%) both on CEUS and CECT/CEMRI. The specificity and accuracy of CEUS in

Table 3 CECT and CEUS enhancement patterns showed in patients with benign lesions

\begin{tabular}{|c|c|c|c|c|c|c|}
\hline \multirow[t]{2}{*}{ Enhancement pattern } & \multicolumn{2}{|c|}{ Typical } & \multicolumn{2}{|c|}{ Atypical } & \multicolumn{2}{|l|}{ Total } \\
\hline & $\overline{\mathrm{CECT}}$ & $\overline{\text { CEUS }}$ & $\overline{\text { CECT }}$ & CEUS & $\overline{\text { CECT }}$ & CEUS \\
\hline Hemangiomas & 16 & 10 & 0 & 6 & 16 & 16 \\
\hline Adenomas & 2 & 2 & 0 & 0 & 2 & 2 \\
\hline Regenerating nodules & 2 & 2 & 0 & 0 & 2 & 2 \\
\hline Hydatid cysts & 2 & 2 & 0 & 0 & 2 & 2 \\
\hline Total & 22 & 16 & 0 & 6 & 22 & 22 \\
\hline
\end{tabular}

detecting tumor vascularity were $98.2 \%$ and $96.6 \%$, respectively. The study concluded that, in the detection of HCC tumor vascularity and assessment of response to thermal ablation, real-time CEUS provided results comparable to those obtained with CT and MRI [21]. These findings are also in agreement with Salvaggio et al. who evaluated the ability of CEUS with second-generation contrast agent in monitoring RFA and TACE treatments of 148 HCCs. In RFA treatment, CEUS showed a sensitivity of $83.3 \%$ and a specificity of $100 \%$ using CT as reference standard with no statistical difference. CEUS detected all cases of incomplete response in HCCs treated with TACE using angiography as reference standard [22].

In this study, no adverse events occurred after SonoVue administration to any of the patients. The safety profile of SonoVue in this study is in agreement with Sporea et al. who used CEUS using SonoVue to evaluate hepatic focal lesions in 294 patients and reported no adverse events in any of their patients [23]. 
The principal limitation of this study was the limited lesion number, mainly due to the exclusion of patients with suboptimal US scan due to the patient body habitus or intervening bowel gas which should be considered a major limitation in the applicability of the technique. In Cantisani et al. study, CEUS still presents the same important drawbacks of every US examination, including operator dependency, obese patients, and non-compliant subjects. For these reasons, if the B-mode US is unsatisfactory, the subsequent CEUS examination will be suboptimal. A specific limitation of CEUS in studying the liver is that limited spatial resolution and, as such, very small lesions may be missed. The US study of the subdiaphragmatic liver by subcostal scanning is sometimes inadequate, especially in patients with a high lying diaphragm [24]. Also, SonoVue role in percutaneous ablation is limited because of its short-lasting enhancement effect and thus, a new second-generation sonographic contrast agent, Sonazoid, with post-vascular phase is more useful as a contrast agent during thermal ablation of HCCs [25]. Sonazoid allows real-time vascular imaging, stable Kupffer phase imaging lasting up to $60 \mathrm{~min}$ (which is not possible with SonoVue), its use is tolerable for multiple scanning and enables the detection of Bmode ill-defined nodules, facilitating correct staging of HCC before treatment [26].

\section{Conclusion}

CEUS is an effective tool in characterization of hepatic focal lesions and recommended as a second diagnostic step after conventional ultrasound to immediately establish the diagnosis especially for benign lesions avoiding further and more expensive examinations and also should be a valuable alternative when a contrast study is needed and CT and MRI contrast agents are contraindicated, as in patients with renal failure and patients with known allergic reaction to CT/MRI contrast agents. Also, CEUS could be used in patients who need shortterm interval repeated regular follow-up by triphasic liver CT in whom, we can do CEUS and CECT alternately and consequently reduce the patient exposure to ionizing radiation and to iodine-based contrast agents.

\section{Abbreviations}

CT: Computed tomography; MRI: Magnetic resonance imaging; PET: Positron emission tomography; HFLs: Hepatic focal lesions; CEUS: Contrast-enhanced ultrasound; CECT: Contrast-enhanced computed tomography; CEMRI: Contrast-enhanced magnetic resonance imaging; MHz: Megahertz; Hz: Hertz; HCC: Hepatocellular carcinoma; AFP: Alpha fetoprotein; PVT: Portal vein thrombosis; RFA: Radiofrequency ablation; TACE: Trans-arterial chemoembolization

\section{Acknowledgements}

Not applicable

\section{Authors' contributions}

MG carried out the CEUS studies and HM participated in the design of the study. MH collected the data and participated in the study design. The first draft of the manuscript was written by SA. All authors read and approved the final manuscript.

Funding

This work has not received any funding.

\section{Availability of data and materials}

All data generated or analyzed during this study are included in this article.

\section{Ethics approval and consent to participate}

The study protocol was approved by the Ethical Committee of Ain Shams University (reference number is not applicable), and written informed consent was obtained from all patients to participate in the study.

\section{Consent for publication}

Written informed consent was obtained from all patients for publication of the study.

\section{Competing interests}

The authors declare that they have no competing interests.

\section{Author details}

${ }^{1}$ Radiodiagnosis Department, Ain Shams University, Cairo, Egypt.

${ }^{2}$ Radiodiagnosis Department, Theodor Bilharz Research Institute, Giza, Egypt.

Received: 19 October 2020 Accepted: 29 November 2020

Published online: 05 January 2021

References

1. Vilgrain V, Boulos L, Vullierme MP, Denys A, Terris B, Menu Y (2000) Imaging of atypical hemangiomasof the liver with pathologic correlation. Radio Graphics 20:379-397

2. González-Añón M, Cervera-Deval J, García-Vila JH et al (1999) Characterization of solid liver lesionswith color and pulsed Doppler imaging. Abdom Imaging 24:137-143

3. Chung YE, Kim MJ, Kim YE, Park MS et al (2013) Characterization of incidental liver lesions: comparison of multidetector $C T$ versus Gd-EOBDTPA-Enhanced MR Imaging. Plos One 8(6):e66141

4. Ali SA, AbdElkhalek YI (2016) Value of integrated PET/CT in detection of hepatic metastatic deposits. EJRNM 47(2):459-465

5. Mansour MG, Ali SA (2016) Transarterial chemoembolization using drug eluting microspheres in refractory colorectal liver metastases with 18F-FDG PET/CT follow-up to assess therapeutic response. EJRNM 47(4):1467-1472

6. Ali SA, Amin DH, Abdelkhalek YI (2020) Efficiency of whole-body 18F-FDG PET $C T$ in detecting the cause of rising serum AFP level in post-therapeutic follow-up for HCC patients. Japan J Radiol 38(5):472-479

7. Brannigan M, Burns PN, Wilson SR (2004) Blood flowpatterns in focal liver lesions at microbubble-enhancedUS. Radio Graphics 24:921-935

8. Quaia E, D'Onofrio M, Palumbo A, Rossi S, BruniS CM (2006) Comparison of contrast-enhanced ultrasonographyversus baseline ultrasound and contrastenhanced computed tomography in metastaticdisease of the liver: diagnostic performanceand confidence. Eur Radiol 16:1599-1609

9. Strobel D, Seitz K, Blank W et al (2009) Tumor-specificvascularization pattern of liver metastasis, hepatocellularcarcinoma, hemangioma and focal nodularhyperplasia in the differential diagnosis of 1,349liver lesions in contrast-enhanced ultrasound(CEUS). Ultraschall Med 30:376-382

10. Zheng SG, Xu HX, Liu LN (2014) Management of hepatocellular carcinoma: The role of contrast-enhanced ultrasound. World J Radio 16(1):7-14

11. D'Onofrio M, Crosara S, Robertis R, Canestrini S, Mucelli RP (2015) Contrastenhanced ultrasound of focal liver lesions. AJR 205:W56-W66

12. D'Onofrio M, Romanini L, Serra C, Magnolfi F et al (2015) Contrast enhancement ultrasound application in focal liver lesions characterization: a retrospective study about guidelines application (SOCEUS-CEUS survey). J Ultrasound 19(2):99-106

13. Strobel D, Seitz K, Blank W et al (2008) Contrast-enhanced ultrasound for the characterization of focal liver lesions: diagnostic accuracy in clinical practice (DEGUM multicenter trial). Ultraschall Med 29:499-505

14. Tranquart F, Le Gouge A, Correas JM et al (2008) Role of contrast-enhanced ultrasound in the blinded assessment of focal liver lesions in comparison with MDCT and CEMRI: results from a multicentre clinical trial. Eur J Cancer Suppl 6:9-15 
15. Sporea I, Şirli R (2014) Is contrast enhanced ultrasound (CEUS) ready for use in daily practice for evaluation of focal liver lesions? Med Ultrason 16(1):37-40

16. Trillaud H, Bruel JM, Valette PJ, Vilgrain V et al (2009) Characterization of focal liver lesions with SonoVue-enhanced sonography: international multicenter-study in comparison to CT and MRI. World I Gastroenterol 15(30):3748-3756

17. MartieA SI, Popescu A, Şirli R (2011) Contrast enhanced ultrasound for the characterization of hepatocellular carcinoma. Med Ultrason 13(2):108-113

18. Laroia ST, Bawa SS, Jain D (2013) Contrast ultrasound in hepatocellular carcinoma at a tertiary liver center: first Indian experience. World J Radiol 5(6):229-240

19. Rossi S, Ghittoni G, Ravetta V, Viera FT, Rosa L et al (2008) Contrastenhanced ultrasonography and spiral computed tomography in the detection and characterization of portal vein thrombosis complicating hepatocellular carcinoma. Eur Radiol 18(8):1749-1756

20. Sorrentino P, D'Angelo S, Tarantino L, Ferbo U, Bracigliano A, Vecchione R (2009) Contrast-enhanced sonography versus biopsy for the differential diagnosis of thrombosis in hepatocellular carcinoma patients. World J Gastroenterol 15(18):2245-2251

21. Lu MD, Yu XL, Li AH, Jiang TA et al (2007) Comparison of contrast enhanced ultrasound and contrast enhanced CT or MRI in monitoring percutaneous thermal ablation procedure in patients with hepatocellular carcinoma: a multicenter study in China. Ultrasound Med Biol 33(11):1736-1749

22. Salvaggio G, Campisi A, Lo Greco V, Cannella I, Meloni MF, Caruso G (2010) Evaluation of posttreatment response of hepatocellular carcinoma: comparison of ultrasonography with second-generation ultrasound contrast agent and multidetector CT. Abdom Imaging 35(4):447-453

23. Sporea I, Sirli R, Martie A, Popescu A, Danila M (2010) How useful is contrast enhanced ultrasonography for the characterization of focal liver lesions? J Gastrointestin Liver Dis 19(4):393-398

24. Cantisani V, Grazhdani H, Fioravanti C et al (2014) Liver metastases: Contrastenhanced ultrasound compared with computed tomography and magnetic resonance. World J Gastroenterol 20(29):9998-10007

25. Masuzaki R, Shiina S, Tateishi R, Yoshida H, Goto E et al (2011) Utility of contrast-enhanced ultrasonography with Sonazoid in radiofrequency ablation for hepatocellular carcinoma. J Gastroenterol Hepatol 26:759-764

26. Kudo M (2016) Defect reperfusion imaging with sonazoid: a breakthrough in hepatocellular carcinoma. Liver Cancer 5(1):1-7

\section{Publisher's Note}

Springer Nature remains neutral with regard to jurisdictional claims in published maps and institutional affiliations.

\section{Submit your manuscript to a SpringerOpen ${ }^{\circ}$ journal and benefit from:}

- Convenient online submission

- Rigorous peer review

- Open access: articles freely available online

- High visibility within the field

- Retaining the copyright to your article

Submit your next manuscript at $\boldsymbol{\nabla}$ springeropen.com 